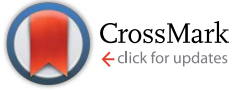

Cite this: J. Mater. Chem. A, 2015, 3, 11631

Received 22nd April 2015 Accepted 29th April 2015

DOI: $10.1039 / c 5 t a 02950 c$

www.rsc.org/MaterialsA

\section{Tin perovskite/fullerene planar layer photovoltaics: improving the efficiency and stability of lead-free devices $\uparrow$}

\begin{abstract}
Kenneth P. Marshall, Richard I. Walton and Ross A. Hatton*
We report the first demonstration of orthorhombic $\mathrm{CsSnl}_{3}$ films prepared from solution at room temperature that have defect densities low enough for use as the light harvesting semiconductor in photovoltaic devices even without using excess $\mathrm{Sn}$ in the preparative method, and demonstrate their utility in a model $\mathrm{p}-\mathrm{i}-\mathrm{n}$ photovoltaic device based on a Cul $\left|\mathrm{CsSnl}_{3}\right|$ fullerene planar layer architecture. We also report an effective strategy for simultaneously improving both the efficiency and stability of these devices towards air exposure based on the use of excess of $\mathrm{Snl}_{2}$ during $\mathrm{CsSnl}_{3}$ synthesis from $\mathrm{Csl}$ and $\mathrm{Snl}_{2}$. A combination of photoelectron spectroscopy, contact potential measurements and device based studies are used to elucidate the basis for this improvement and role of the excess $\mathrm{Snl}_{2}$. The open-circuit voltage in these lead-free photovoltaic devices is shown to be strongly dependent on the degree of alignment between the perovskite conduction band edge and the lowest occupied molecular orbital (LUMO) in the fullerene electron transport layer. Furthermore, the energetics at the perovskitefullerene interface are shown to be a function both of the LUMO energy of the fullerene and the nature of the interaction at the heterojunction which can give rise to a large abrupt vacuum level shift across the interface. A champion open-circuit voltage of $\sim 0.55 \mathrm{~V}$ is achieved using indene- $\mathrm{C}_{60}$ bis-adduct as the electron extraction layer, which is twice that previously reported for a $\mathrm{CsSnl}_{3}$ based PPV.
\end{abstract}

\section{Introduction}

Over the past 4 years there has been dramatic progress in the field of perovskite photovoltaics (PPVs), most of which has focused on using methylammonium (MA) lead iodide $\left(\mathrm{MAPbI}_{3}\right)^{1-4}$ and chloride substituted analogues, $\mathrm{MAPbI}_{3-x} \mathrm{Cl}_{x},{ }^{5-7}$ as the light harvesting semiconductor. This is due to the high absorption coefficient, high charge carrier diffusion length $\left(L_{\mathrm{D}}>\right.$ $100 \mathrm{~nm})^{8}$ and low band gap $\left(E_{\mathrm{g}}\right)$ of $1.5-1.6 \mathrm{eV}^{5,9}$ in these materials. The highest power conversion efficiency $(\eta)$ achieved to date in this field is over $19 \% .^{\mathbf{1 0}}$

The majority of PPV devices reported to date use the perovskite in a distributed heterojunction device architecture, in which n-type mesoporous $\mathrm{TiO}_{2}$ serves as the electron-transport layer (ETL) and a p-type organic semiconductor is used as the hole-transport layer (HTL). There are also a smaller number of reports of planar-heterojunction PPVs based on a $\mathrm{p}-\mathrm{i}-\mathrm{n}$ structure, including those in which $\mathrm{TiO}_{2}$ is replaced with organic semiconductors such as fullerenes, which have achieved $\eta>$ 17\%. ${ }^{11-13}$ Unlike $\mathrm{TiO}_{2}$, fullerene ETLs do not require annealing which removes the need for the substrate electrode to be able to

Department of Chemistry, University of Warwick, Coventry, CV4 7AL, UK. E-mail: ross. hatton@warwick.ac.uk

$\dagger$ Electronic supplementary information (ESI) available: Further characterisation data. See DOI: $10.1039 / \mathrm{c} 5$ ta02950c withstand high temperatures. Planar device architectures using organic charge transport layers may also offer the best prospects for accommodating the very large thermal expansion exhibited by some metal halide perovskites. ${ }^{\mathbf{1 4}}$ There is also interest in identifying low cost organic and inorganic HTLs, most recently the $\mathrm{Cu}(\mathrm{I})$ compounds $\mathrm{CuI}$ and CuSCN. ${ }^{15-18}$

The primary drawback of $\mathrm{Pb}$ perovskites for photovoltaic applications is their susceptibility to degradation in the presence of water to produce $\mathrm{PbI}_{2},{ }^{19-21}$ which is a partially water soluble compound. ${ }^{21,22}$ Soluble $\mathrm{Pb}$ compounds are well known to be toxic to both humans and the natural environment ${ }^{23}$ and so there is growing interest in the development of alternative, narrow $E_{\mathrm{g}}$ perovskites that use $\mathrm{Sn}$ instead of $\mathrm{Pb},{ }^{24}$ such as $\mathrm{CsSnI}_{3}{ }^{25-28}$ and $\mathrm{MASnI}_{3} \cdot{ }^{29,30}$

At room temperature and in the absence of air $\mathrm{CsSnI}_{3}$ adopts a 3D orthorhombic perovskite structure which is known as the black phase. The $E_{\mathrm{g}}$ of this semiconductor is near optimal for PV applications $\left(\sim 1.3 \mathrm{eV}^{\mathbf{1 4}}\right),{ }^{31}$ which combined with its high optical absorption coefficient $\left(\sim 10^{4} \mathrm{~cm}^{-1} \text { in the visible region }\right)^{32}$ and low exciton binding energy $(\sim 18 \mathrm{meV}),{ }^{33}$ makes $\mathrm{CsSnI}_{3}$ attractive as a light harvesting semiconductor in PPVs because photoexcitation results in free carrier generation for light with wavelengths as long as $\sim 950 \mathrm{~nm} .{ }^{14}$ However, the material is prone to the formation of a high density of Sn cation vacancy defects, which serve as p-type dopants and recombination centres. ${ }^{\mathbf{1 4 , 3 4}}$ 
Sn perovskites also have the drawback of air sensitivity, with the black phase converting to yellow phase $\mathrm{CsSnI}_{3}$, which in turn oxidises to $\mathrm{Cs}_{2} \mathrm{SnI}_{6}$ in which $\mathrm{Sn}$ is in its more stable 4+ oxidation state. ${ }^{14,35}$ Unlike $\mathrm{Sn}, \mathrm{Pb}$ is most stable in the $2+$ oxidation state due to the relativistic lowering of the Bohr radius of s-orbitals more than that of other atomic orbitals, which stabilises them towards oxidation, an effect that is most pronounced in heavy p-block elements like $\mathrm{Pb} .{ }^{36,37}$ In practice the ingress of oxygen and moisture into PV devices is inevitable and so a central challenge in Sn-PPV research is to develop strategies that can be used in conjunction with device encapsulation to reduce the rate of oxidation of $\mathrm{Sn}$ perovskite enough for practical applications to be possible. To date there have been six literature reports relating to the use of Sn perovskites in PPVs which collectively demonstrate the potential to achieve high $\eta .^{25-30}$

$\mathrm{CsSnI}_{3}$ was first used by Chung et al. ${ }^{25}$ as a HTL in PPVs in which a ruthenium dye was the primary light harvesting element and the $\mathrm{CsSnI}_{3}$ was synthesised using a vacuum melt process at $450{ }^{\circ} \mathrm{C}$. That pioneering work was quickly followed by Chen et al. ${ }^{26}$ who demonstrated a Schottky contact type PPV based on a vacuum evaporated layer of $\mathrm{CsSnI}_{3}$ formed by sequential deposition of alternating layers of CsI and $\mathrm{SnCl}_{2}$ followed by annealing at $175{ }^{\circ} \mathrm{C}$ to form the perovskite, although the device fill factor $(\mathrm{FF})$ was extremely low $(\sim 0.22)$ due to a very low shunt resistance and high series resistance. Concurrently to this Zhou et al. ${ }^{38}$ reported that $\mathrm{CsSnI}_{3}$ could be synthesised at room temperature from a mixed solution of CsI and $\mathrm{SnI}_{2}$, although films formed using that method were not tested for optoelectronic applications. In 2014 Noel et al. reported the highest $\eta$ to date for a Sn PPV of $\sim 6 \%$, achieved using MASnI $_{3}$ as the light harvesting semiconductor. ${ }^{29}$ However those devices were also shown to exhibit a very large variation in performance and poor stability with deeply coloured devices becoming completely transparent minutes after measuring. ${ }^{29}$ That work was published only days before a report by Hao et al. ${ }^{\mathbf{3 0}}$ who showed that $\eta$ close to $6 \%$ could be achieved using $\mathrm{MASnIBr}_{2}$ and the open-circuit voltage $\left(V_{\mathrm{oc}}\right)$ could be tuned by varying the ratio of iodide to bromide as a result of a change in $E_{\mathrm{g}}$. Shortly afterwards Kumar et al. ${ }^{27}$ reported that $\mathrm{CsSnI}_{3}$ processed at $70{ }^{\circ} \mathrm{C}$ and with $20 \mathrm{~mol} \% \mathrm{SnF}_{2}$ additive could be used as the light absorber in a PPV device based on a distributed heterojunction with $\mathrm{TiO}_{2}$, achieving photocurrents $>22 \mathrm{~mA}$ $\mathrm{cm}^{-2}$. Unfortunately the $V_{\mathrm{oc}}$ and fill factor (FF) were limited to $\sim 240 \mathrm{mV}$ and $\sim 0.37$ respectively which constrained $\eta$ to $2.02 \%$. Notably, devices fabricated without $\mathrm{SnF}_{2}$ did not function and whilst it was shown that fluoride was not incorporated into the $\mathrm{CsSnI}_{3}$ lattice, the possible optoelectronic functionality of the relatively large quantity of $\mathrm{SnF}_{2}$ present in the perovskite film was not discussed. ${ }^{27}$ Kumar et al. concluded that the $V_{\mathrm{oc}}$ in $\mathrm{CsSnI}_{3}$ PVs is limited by the high background carrier density due to Sn-vacancy defects even when prepared under Sn-rich conditions, and is not set by the difference between the conduction band minimum of the ETL and the valence band maximum (or highest occupied molecular orbital (HOMO)) of the HTL. The same group went on to show that substitution of iodide by bromide in $\mathrm{CsSnI}_{3}$ is an effective means of increasing the $V_{\text {oc }}$ in Sn-PPV achieving a $V_{\text {oc }}$ of $0.41 \mathrm{~V}$. However, the improvement in $V_{\mathrm{oc}}$ was at the expense of photocurrent due to the increase in $E_{\mathrm{g}}{ }^{28}$

Herein we report: (i) the first demonstration of orthorhombic $\mathrm{CsSnI}_{3}$ films prepared from solution at room temperature with defect densities low enough for use as the light harvesting semiconductor in PPV devices, even in the absence of excess Sn; (ii) a new strategy for improving device stability towards oxidation in air, which does not complicate the process of device fabrication; (iii) the results of a study which unambiguously show that the energetics at the interface between $\mathrm{CsSnI}_{3}$ and ETL are a key determinant of device $V_{\text {oc }}$.

\section{Results}

Model p-i-n PPVs were fabricated with the structure: indium tin oxide (ITO) $|\mathrm{CuI}| \mathrm{CsSnI}_{3} \mid$ fullerene|bathocuproine (BCP)|Al, as schematically illustrated in Fig. 1a. The $\mathrm{CsSnI}_{3}$ layer was prepared from $8 \mathrm{wt} \% \mathrm{CsSnI}_{3}$ in $\mathrm{N}, \mathrm{N}$-dimethylformamide (DMF) using $\mathrm{SnI}_{2}$ and CsI. CuI was chosen as the p-type material due to its proven effectiveness as a HTL in PPVs. ${ }^{15} \mathrm{CuI}$ is also amenable to deposition by both thermal evaporation and by solution processing. Since thermal evaporation enables a very high degree of control over film thickness and morphology it is the preferred method in this study. Likewise $\mathrm{C}_{60}$ was selected as the n-type material because it can also be deposited by thermal evaporation. There are also a number of simple derivatives of $\mathrm{C}_{60}$ commercially available with modified frontier orbital energies. For these reasons $\mathrm{C}_{60}$ serves as a model n-type charge extraction layer in this work. The $\mathrm{CsSnI}_{3}$ layer was deposited by spin casting directly onto freshly evaporated $\mathrm{CuI}$ at room temperature to give films with a thickness of $\sim 50 \mathrm{~nm}$, as measured using cross-sectional AFM histogram analysis (Fig. 1b(ii)). In contrast to all other reports relating to the use of Sn-perovskites in PPVs these films were used without postdeposition annealing.

Fig. $1 \mathrm{~b}(\mathrm{i})$ shows a representative SEM image of a $\mathrm{CsSnI}_{3}$ film deposited from $8 \mathrm{wt} \% \mathrm{CsSnI}_{3}$ in DMF onto a CuI coated ITO substrate. The film is made up of small crystals approximately 50-100 $\mathrm{nm}$ in diameter, which is comparable to the film thickness determined from a step edge analysis using atomic force microscopy (Fig. 1b(ii) and (ESI) Fig. S1a-c $\dagger$ ). It is therefore likely that these crystallites extend from the top to the bottom of the film as schematically depicted in Fig. 1a. Increasing the loading of $\mathrm{CsSnI}_{3}$ to $15 \mathrm{wt} \%$ resulted in an increase in film thickness to $\sim 80 \mathrm{~nm}$ with a larger mean crystallite size and very few pin holes (Fig. 1c and ESI Fig. S1d-f $\dagger$ ). Whilst there are still some gaps in films of this thickness the coverage is exceptionally high for such a thin layer of metal halide perovskite. ${ }^{11}$ Furthermore, films of comparable quality could also be prepared on glass, ITO and Au coated substrates which proved useful for fundamental measurements of film properties.

A representative X-ray diffraction (XRD) pattern of a $\mathrm{CsSnI}_{3}$ film spin cast onto a glass substrate pre-coated with $10 \mathrm{~nm}$ of evaporated CuI is shown in Fig. 2b. The very thin CuI layer is included to ensure the structure of the perovskite film probed is identical to that used in PPV devices. Since there is no evidence 


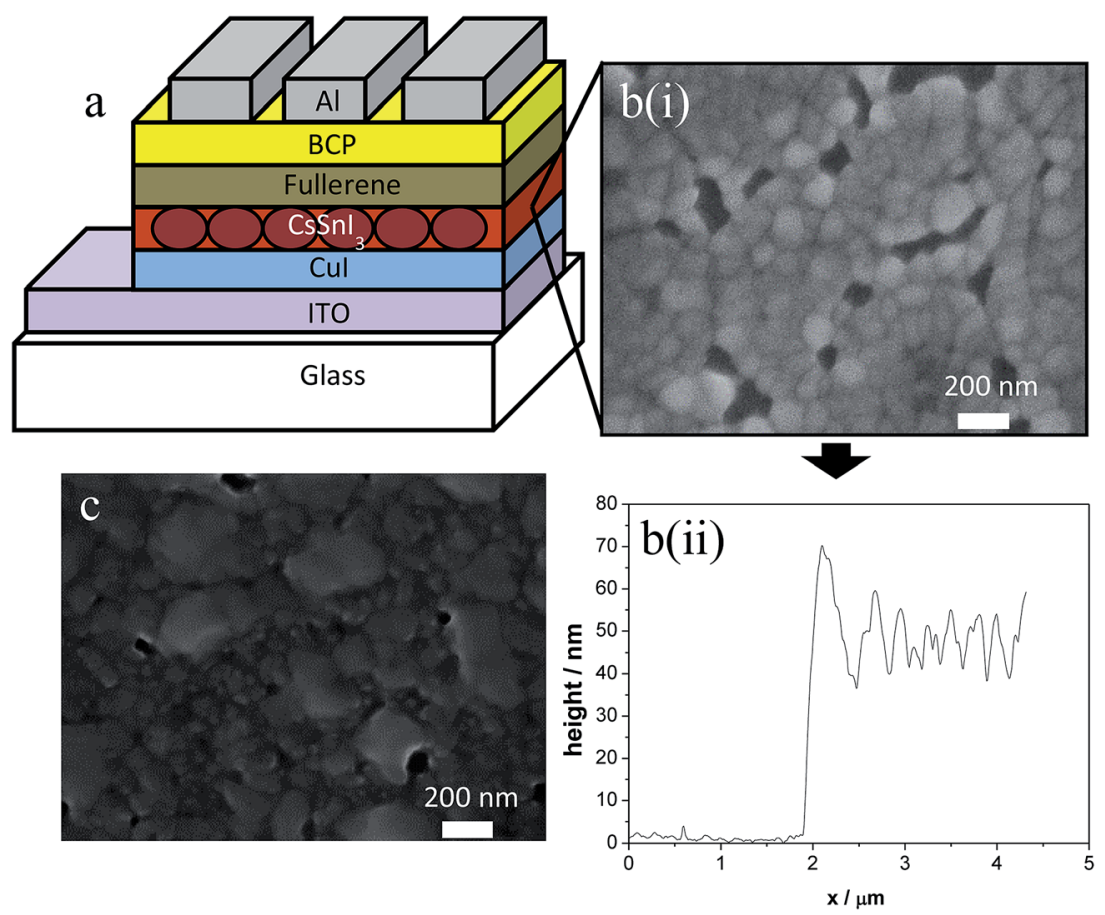

Fig. 1 (a) Schematic of the device architecture used in this work; (b(i)) SEM image of a $\mathrm{CsSnl}_{3}$ film prepared with a 10 mol\% excess Snl 2 and spin cast at $4000 \mathrm{rpm}$ from $8 \mathrm{wt} \%$ solution onto an ITO glass substrate coated with a $100 \mathrm{~nm}$ layer of Cul; (b(ii)) AFM step height of a scored CsSnl 3 film spin cast from $8 \mathrm{wt} \%$ solution onto glass; (c) SEM image of a CsSnl 3 film spun from $15 \mathrm{wt} \% \mathrm{CsSnl}_{3}$ solution with $10 \mathrm{~mol}^{2} \mathrm{Snl}_{2}$ excess using the same deposition process and substrate as for (b(i)).

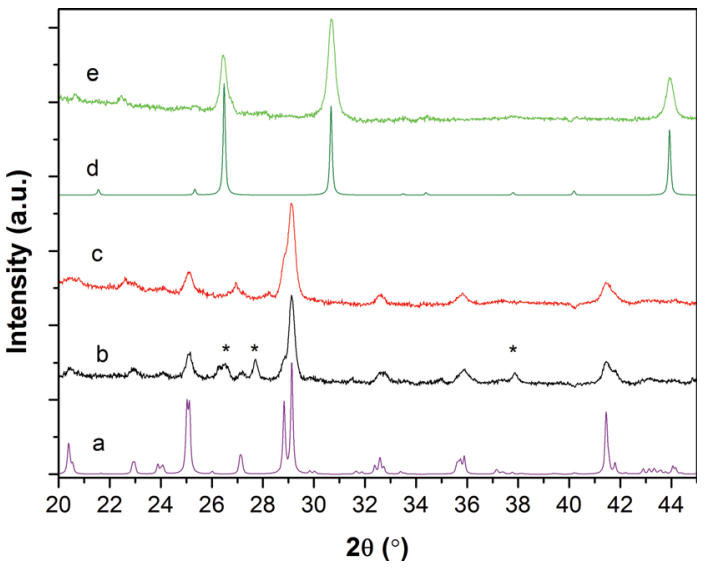

Fig. 2 X-ray diffraction (XRD) patterns of films of: (a) a simulated powder diffraction pattern of $\mathrm{CsSnl}_{3}$; (b) a thin film of $\mathrm{CsSnl}_{3}$; (c) a thin film of $\mathrm{CsSnl}_{3}$ prepared with a $10 \mathrm{~mol} \%$ excess $\mathrm{Snl}_{2}$; (d) a simulated powder diffraction pattern of $\mathrm{Cs}_{2} \mathrm{Snl}_{6}$, and (e) a $\mathrm{CsSnl}_{3}$ film oxidised in air for 16 hours. All films probed using XRD were supported on glass substrates coated with $10 \mathrm{~nm}$ Cul and deposited by spin casting at $2000 \mathrm{rpm}$ from 16 mol\% DMF solutions.

of $\mathrm{CuI}$ in the XRD pattern it can be concluded that the buried CuI layer is essentially amorphous and/or too thin to be observed using this technique. The XRD pattern in Fig. $2 \mathrm{~b}$ is consistent with the orthorhombic perovskite structure expected for the black phase of $\mathrm{CsSnI}_{3}$, as reported in the literature ${ }^{\mathbf{1 4}}$ and simulated in Fig. 2a. The prominence of the peaks at $\sim 29^{\circ}$ in the XRD pattern of the film as compared to the simulated powder pattern is indicative of preferential crystal orientation in the spin cast film on CuI. The small reflections at $\sim 26.3^{\circ}, \sim 27.7^{\circ}$ and $\sim 37.8^{\circ}$ (starred) are associated with the supporting substrate and sample holder. After 16 hours air exposure the $\mathrm{CsSnI}_{3}$ film gives the XRD pattern Fig. 2e, which is consistent with the formation of the $\mathrm{Cs}_{2} \mathrm{SnI}_{6}$, in which $\mathrm{Sn}$ is in the more stable 4+ oxidation state, as is evident from the good agreement with the simulated reflections for $\mathrm{Cs}_{2} \mathrm{SnI}_{6}$ shown in Fig. $2 \mathrm{~d} .{ }^{39} \mathrm{X}$ ray photoelectron spectroscopy (XPS) measurements of the oxygen 1s binding energy shows that those Sn atoms that do not form part of $\mathrm{Cs}_{2} \mathrm{SnI}_{6}$ structure are most likely present as $\mathrm{SnO}_{2}$ (ESI Fig. S2 $\dagger$ ). The absence of reflections in the XRD pattern indicative of crystalline $\mathrm{SnO}_{2}$ indicates that it is present as an amorphous or nano-crystalline phase.

It has recently been proposed that due to the ease with which $\mathrm{Sn}$-cation vacancies form in $\mathrm{CsSnI}_{3}$ it must be prepared in a Snrich environment or at high temperature to ensure the density of these defects is low enough for its use as a light harvesting material in PPV devices. ${ }^{27,34,35}$ However we find that devices based on $\mathrm{CsSnI}_{3}$ films prepared using stoichiometric mixtures of CsI and $\mathrm{SnI}_{2}$ with no excess of $\mathrm{Sn}$ and processed entirely at room temperature generate a short circuit current density $\left(J_{\mathrm{sc}}\right)$ of $\sim 10 \mathrm{~mA} \mathrm{~cm}{ }^{-2}$ under 1 sun simulated solar illumination, which is a very high current for such a thin semiconductor layer ( $\sim 50 \mathrm{~nm})$ (Fig. 3). The incident-photon-to-converted-electron (IPCE) efficiency (ESI Fig. $3 \dagger$ ) confirms that $\mathrm{CsSnI}_{3}$ is the primary light absorbing component in these devices, since wavelengths as long as $\sim 930 \mathrm{~nm}$ are converted into electrons in the external circuit, which corresponds to sub-band gap photon 

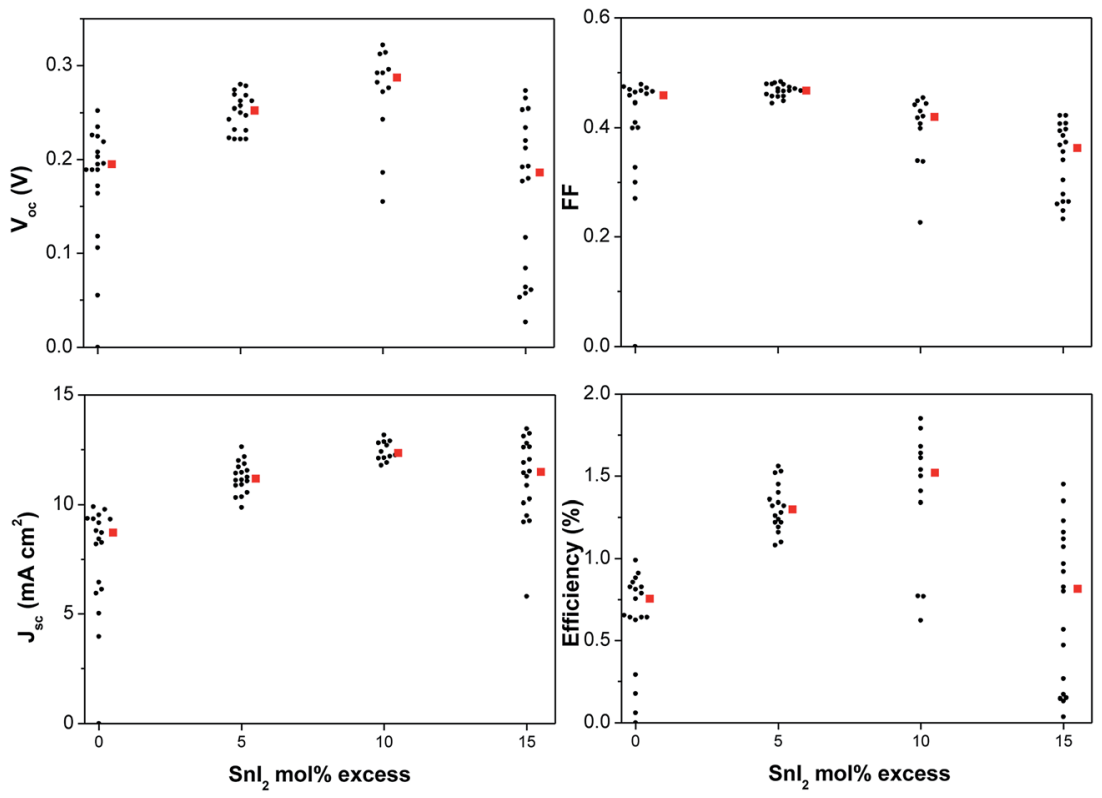

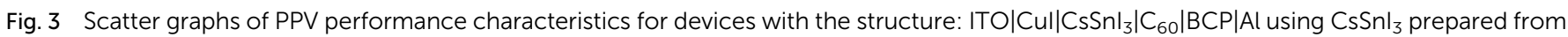
solutions containing either 0,5 or 10 mol\% excess $\mathrm{Snl}_{2}$. The mean values are shown as red squares.

energies for $\mathrm{C}_{60}$. This spectral response is also consistent with $E_{\mathrm{g}}$ for $\mathrm{CsSnI}_{3}$ reported in the literature of $\sim 1.3 \mathrm{eV} .^{14,35}$

As shown in Fig. 3, devices based on $\mathrm{CsSnI}_{3}$ films prepared using an excess of $\mathrm{SnI}_{2}$ and $\mathrm{C}_{60}$ ETL exhibit up to $\sim 30 \%$ increase in $J_{\mathrm{sc}}$ and $V_{\mathrm{oc}}$ as compared to those without. The optimal $\mathrm{SnI}_{2}$ excess is $\sim 10 \mathrm{~mol} \%$ which results in a doubling of $\eta$ from $\sim 0.75 \%$ to $\sim 1.5 \%$. All reflections in the XRD pattern for films prepared using $10 \mathrm{~mol} \%$ excess $\mathrm{SnI}_{2}$ (Fig. 2c) can be attributed to $\mathrm{CsSnI}_{3}$, except the weak reflection at $\sim 28.5^{\circ}$. The latter cannot be assigned to $\mathrm{SnI}_{2}$ or its oxidised form; $\mathrm{SnI}_{4}$, which indicates that the excess $\mathrm{SnI}_{2}$ is nano-crystalline or amorphous. High-resolution XPS measurements of the surface chemical composition of freshly prepared films with a $10 \mathrm{~mol} \% \mathrm{SnI}_{2}$ excess show that there is very little $\mathrm{SnI}_{2}$ and no evidence for $\mathrm{SnI}_{4}$ at the $\mathrm{CsSnI}_{3}$ surface (Fig. S4 $\dagger$ ), since $\sim 95 \%$ of the photoelectrons originate from the top $\sim 7 \mathrm{~nm}$ of the sample. The XPS data therefore provides evidence that the $10 \mathrm{~mol} \%$ excess $\mathrm{SnI}_{2}$ phase separates to the buried interface upon solvent evaporation. Notably, since these films were unavoidably exposed to the laboratory for $\sim 1$ minute when transferring from the nitrogen filled glove box to the spectrometer vacuum system there is also evidence for significant oxidation of the surface of the $\mathrm{CsSnI}_{3}$ to $\mathrm{Cs}_{2} \mathrm{SnI}_{6}$ in the XPS spectra.

If the excess $\mathrm{SnI}_{2}$ is primarily located at the buried $\mathrm{CsSnI}_{3} \mid \mathrm{CuI}$ interface it must be capable of transporting holes from $\mathrm{CsSnI}_{3}$ into CuI since PPV devices incorporating up to $15 \mathrm{~mol} \% \mathrm{SnI}_{2}$ perform comparably to those with $5 \% \mathrm{SnI}_{2}$ excess (Fig. 3). To our knowledge there are no previous reports of $\mathrm{SnI}_{2}$ being used as a semiconductor in an organic or hybrid electronic device and so its charge transport properties are not known. To investigate the transport properties of $\mathrm{SnI}_{2}$ we have estimated the hole-mobility in $\mathrm{SnI}_{2}$ from the space charge limited current in unipolar diodes to be $2.2-5.1 \times 10^{-6} \mathrm{~cm}^{2} \mathrm{~V}^{-1} \mathrm{~s}^{-1}$ (ESI, Fig. S5 $\dagger$ ). Whilst this value is low as compared to that of $\mathrm{Sn}$ and $\mathrm{Pb}$ halide perovskites it is comparable to that of many amorphous organic semiconductors ${ }^{40}$ and offers an explanation as to why device $\mathrm{FF}$ begins to deteriorate with increasing $\mathrm{SnI}_{2}$ excess beyond $10 \mathrm{~mol} \%$. To determine the accessibility of the $\mathrm{SnI}_{2}$ valence band for hole transport we have measured the ionisation potential $\left(I_{\mathrm{p}}\right)$ of $\mathrm{SnI}_{2}\left(I_{\mathrm{p}} \sim 5.64 \mathrm{eV} \pm 0.05\right.$ $\mathrm{eV})$ and CuI films $\left(I_{\mathrm{p}} \sim 5.84 \mathrm{eV} \pm 0.05 \mathrm{eV}\right)$ using ultraviolet photoelectron spectroscopy (UPS). Full details of the UPS study are given in the (ESI Fig. S6 and S7†). These energy levels, along with the $E_{g}$ for $\mathrm{CuI}$ determined from electronic absorption measurements (ESI, Fig. S8 $\dagger$ ) and $E_{\mathrm{g}}$ for $\mathrm{SnI}_{2}$ taken from the literature, ${ }^{41}$ are given in Fig. 4a. The susceptibility of $\mathrm{CsSnI}_{3}$ films to oxidation in air prevented us from making a reliable measurement of the $\mathrm{CsSnI}_{3}$ $I_{\mathrm{p}}$ using UPS, and the values reported in the literature for argon sputtered $\mathrm{CsSnI}_{3}$ - also measured using UPS - vary from $4.9 \mathrm{eV}^{25}$ to $5.7 \mathrm{eV} .^{41}$ In order to estimate the position of the valence and conduction band edges in $\mathrm{CsSnI}_{3}$ with confidence we have measured the work function of $\mathrm{CsSnI}_{3}$ films without air exposure, or argon sputtering, using a Kelvin probe located in the same glove box as the spin coater used to deposit the perovskite film. On the basis of this measurement, combined with the known $E_{\mathrm{g}}$ $(\sim 1.3 \mathrm{eV})$ and knowledge of the tendency of this material to form p-type defects, ${ }^{27,34,35}$ we have estimated the possible range of energies for the valence and conduction band edges in $\mathrm{CsSnI}_{3}$. These energy levels along with the work function of each material measured using the Kelvin probe technique are given in Fig. 4a. It is clear from the location of the Fermi level in CuI that it is heavy p-doped which is consistent with literature reports ${ }^{43}$ and can be attributed to $\mathrm{Cu}$ atom array vacancies. In the absence of such intrinsic defects CuI would block the extraction of holes from the $\mathrm{CsSnI}_{3}$ valance band due to its very deep lying valance band. However, the high density of electron acceptor states near to the valence band edge should ensure that there is no barrier to hole- 
extraction from $\mathrm{CsSnI}_{3}$ when thermodynamic equilibrium is established across the interface. Similarly, spontaneous electron transfer from the $\mathrm{SnI}_{2}$ into $\mathrm{CuI}$ due to the difference in Fermi level would be expected to remove the relatively small barrier to holeextraction from $\mathrm{SnI}_{2}$ into CuI. On the basis of the energy levels given in Fig. 4a there would however be expected to be a significant barrier to hole-transport from the $\mathrm{CsSnI}_{3}$ valence band into the $\mathrm{SnI}_{2}$ valence band. However, since the current-voltage characteristics of PPV devices fabricated with an excess of $\mathrm{SnI}_{2}$ in the $\mathrm{CsSnI}_{3}$ layer (up to $15 \mathrm{~mol} \%$ ) are not consistent with a high device series resistance it is likely that there is a favourable vacuum level shift at the interface due to the formation of an interfacial dipole.

The improvement in $J_{\mathrm{sc}}$ and $V_{\mathrm{oc}}$ in PPV devices using a $\mathrm{CsSnI}_{3}$ layer prepared with excess $\mathrm{SnI}_{2}$ can be rationalised in terms of a reduction in the background carrier density which gives rise to recombination loses, since it is known that the density of Snvacancy defects (i.e. the primary source of the background carrier density) is suppressed when the perovskite is synthesised in a Sn-rich environment. ${ }^{27,34,35}$ However, to investigate the possibility that the energetics at the perovskite $\mid \mathrm{C}_{60}$ interface might also plays a role in controlling $V_{\mathrm{oc}}$ we have measured the change in energy between the perovskite Fermi level and the surface vacuum level (vac) as a function of the $\mathrm{C}_{60}$ layer thickness using the Kelvin probe method. This energy difference, $\varepsilon_{\mathrm{f}}^{\mathrm{vac}}$, corresponds to the work function of $\mathrm{C}_{60}$ when the film thickness is sufficient for the establishment of Fermi level alignment across the interface. If thermodynamic equilibrium is not achieved then $\varepsilon_{\mathrm{f}}^{\mathrm{vac}}$ corresponds to the work function of the perovskite with a modified surface potential. ${ }^{42,43}$ Changes in $\varepsilon_{\mathrm{f}}^{\mathrm{vac}}$ can therefore be interpreted in terms of a vacuum level shift which give rises to a comparable shift in the energy of the HOMO and LUMO in the $\mathrm{C}_{60}$ with respect the valance and conduction band edges in $\mathrm{CsSnI}_{3}$. The results of this experiment are summarised in Fig. 4b. At the interface between $\mathrm{CsSnI}_{3}$ prepared with $0 \%$ excess $\mathrm{SnI}_{2}$ there is no significant change in the energy of the vacuum level. Conversely for films prepared with $10 \mathrm{~mol} \%$ excess $\mathrm{SnI}_{2}$ there is a large abrupt positive vacuum level shift of $\sim 0.3 \mathrm{eV}$ meV. Abrupt positive vacuum level shifts at the interface between inorganic materials and organic semiconductors are relatively rare $^{\mathbf{4 2 , 4 3}}$ and are indicative of ground state charge transfer from the substrate into the first layer of molecule forming an interfacial dipole. The reason for the stronger interaction between $\mathrm{C}_{60}$ and $\mathrm{CsSnI}_{3}$ with fewer Sn-vacancy sites is not yet clear, although the existence of this abrupt positive vacuum level shift offers an alternative explanation for the increase in $V_{\text {oc }}$, since it would operate to reduce the loss in electron potential energy when moving from the perovskite conduction band into the fullerene LUMO.

To further investigate the extent to which $V_{\text {oc }}$ in these devices is controlled by the degree of alignment between the LUMO of the ETL and the conduction band edge in $\mathrm{CsSnI}_{3}$, we have systematically reduced the LUMO energy of the ETL from $\sim 4.00$ $\mathrm{eV}^{45,46}$ to $\sim 3.91 \mathrm{eV}^{46}$ and finally to $\sim 3.74 \mathrm{eV}^{46}$ below the vacuum level by substituting $\mathrm{C}_{60}$ with the $\mathrm{C}_{60}$ derivatives [6,6]phenyl- $\mathrm{C}_{61}$-butyric acid methyl ester $\left(\mathrm{PC}_{60} \mathrm{BM}\right)$ and indene- $\mathrm{C}_{60}$ bis-adduct ( $\left.\mathrm{IC}_{60} \mathrm{BA}\right)$ (Fig. 4a). Whilst the exact magnitude of these values remain the subject of debate ${ }^{45}$ the differences in LUMO energy between these derivatives is firmly established. ${ }^{45,46}$ Champion $J / V$ characteristics in the dark and under 1 sun simulated illumination are given in Fig. 5 and the full data set is given in the ESI (Table S1†). It is clear from Fig. 5 and the full data set in (Table $\mathrm{S} 1 \dagger$ ) that the increase in device $V_{\mathrm{oc}}$ correlates with an increase in the LUMO energy of the fullerene ETL which is consistent with a reduction in the potential energy lost by an electron moving from the $\mathrm{CsSnI}_{3}$ into the ETL as seen in organic PVs. ${ }^{46}$ The highest $\eta$ is achieved for devices using ICBA as the ETL at $2.76 \%$, which is significantly higher than the best previously reported for a $\mathrm{CsSnI}_{3}$ based PPV $(2.02 \%){ }^{27}$ Collectively these data show that the performance of PPVs based on $\mathrm{CsSnI}_{3}$ depends strongly on the energetics at the interface between the $\mathrm{CsSnI}_{3}$ and the ETL, much like that seen at donor/ acceptor heterojunctions in OPVs. ${ }^{46}$

For practical applications the stability of the light harvesting semiconductor towards oxidation in air is critically important. (a)

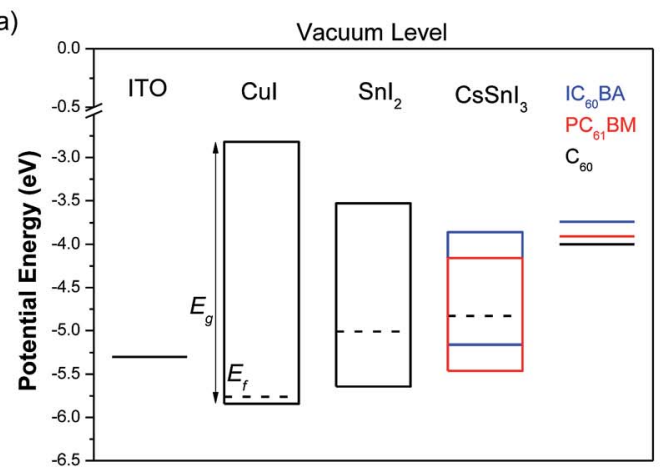

(b)

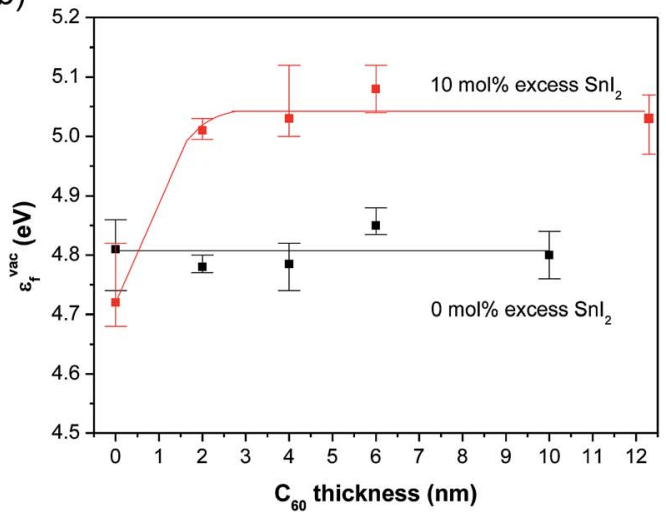

Fig. 4 (a) Schematic energy level diagram depicting the valence band edge for Cul and $\mathrm{Snl}_{2}$ films measured using UPS. The conduction band edge for $\mathrm{Cul}$ has been estimated from the electronic absorption spectrum (ESI, S8 $\dagger$ ). The energy of the conduction band edge for $\mathrm{Snl}_{2}$ is estimated based on the reported $E_{\mathrm{g}}$ for $\mathrm{Snl}_{2} \cdot{ }^{44}$ The work function of freshly UV/O 3 treated ITO glass, Cul, Snl 2 and CsSnl 3 measured using the Kelvin probe technique are also shown; (b) the evolution of $\varepsilon_{f}^{\mathrm{vac}}$ at the surface of a freshly deposited $\mathrm{CsSnl}_{3}$ film with increasing thicknesses of thermally evaporated $\mathrm{C}_{60}$ measured using the Kelvin probe technique. The error bars represent maximum and minimum values. 


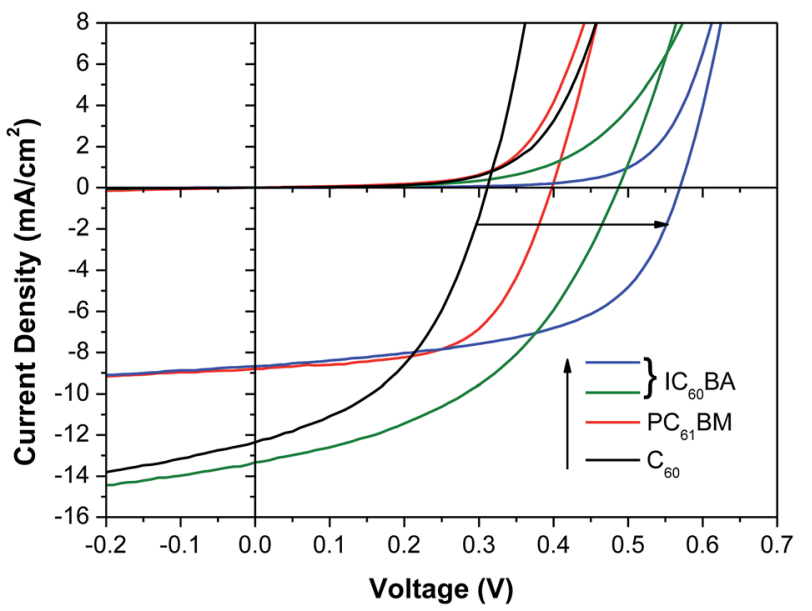

Fig. 5 Dark and light JV curves of best performing PPV devices with the structure ITO|Cul|CsSnl 3 + 10 mol\% Snl $_{2} \mid$ fullerene|BCP|Al, using different fullerene ETLs. For the case of ICBA the device $V_{\text {oc }}$ was found to be strongly dependent on the concentration of the ICBA solution used: $3 \mathrm{mg} \mathrm{ml}^{-1}$ ICBA (green), $5 \mathrm{mg} \mathrm{ml}^{-1}$ ICBA (blue), although was always higher than that achieved in devices using PCBM when all other parts of the device were identical.

Encouragingly PV devices based on $\mathrm{CsSnI}_{3}$ prepared in a Sn-rich environment have been reported to be stable for more than 250 hours when stored in glove box, ${ }^{27}$ although - to our knowledge - the stability of Sn perovskite PVs in air has not be quantitatively reported. We find that the use of excess $\mathrm{SnI}_{2}$ in the preparation of $\mathrm{CsSnI}_{3}$ dramatically improves device stability. Fig. 6 shows the key performance parameters of unencapsulated PPV devices (using $\mathrm{C}_{60}$ as the ETL) as a function of $\mathrm{SnI}_{2}$ excess in the $\mathrm{CsSnI}_{3}$ layer. The data points in the hashed region correspond to device performance immediately after fabrication and after storage for 10 days in a nitrogen filled

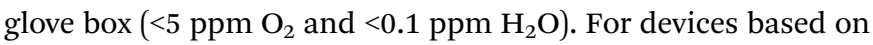
$\mathrm{CsSnI}_{3}$ films prepared using stoichiometric amounts of CsI and $\mathrm{SnI}_{2}$ the $\eta$ of is reduced by $\sim 70 \%$ after this ten day period of storage due to the combined effect of deteriorated $J_{\mathrm{sc}}, V_{\mathrm{oc}}$ and FF. Conversely devices based on $\mathrm{CsSnI}_{3}$ with 10 mol\% excess $\mathrm{SnI}_{2}$ exhibit a relatively small reduction in $\eta$ of $\sim 10 \%$ due primarily to a reduction in FF. These devices were then bought into the laboratory and the performance tested as a function of time. Devices with 0 mol\% excess $\mathrm{SnI}_{2}$ stopped working altogether after $\sim 30$ minutes air exposure due to a complete collapse in $J_{\text {sc }}$. Conversely devices with $10 \mathrm{~mol} \%$ excess $\mathrm{SnI}_{2}$ exhibit dramatically improved stability such that even after 14 hours in air without encapsulation they still function, albeit with a $J_{\text {sc }}$ of one fifth the starting value. Both the FF and $V_{\text {oc }}$ are essentially stable during this period.

It is likely that the deterioration in device performance upon exposure to the laboratory environment results from a number of parallel degradation mechanisms some of which are not related to the perovskite layer, such as oxidation of the $\mathrm{Al}$ cathode and/or doping of the $\mathrm{C}_{60}$ layer by oxygen. ${ }^{47}$ However, since it is evident from our XPS studies (ESI, Fig. S4 $\dagger$ ) that oxidation of $\mathrm{CsSnI}_{3}$ in air to form $\mathrm{Cs}_{2} \mathrm{SnI}_{6}$ is a very rapid process and that $\mathrm{Cs}_{2} \mathrm{SnI}_{6}$ in known to absorb light very weakly as compared to $\mathrm{CsSnI}_{3},{ }^{41}$ it is likely that conversion of $\mathrm{CsSnI}_{3}$ into $\mathrm{Cs}_{2} \mathrm{SnI}_{6}$ is major contributor to the
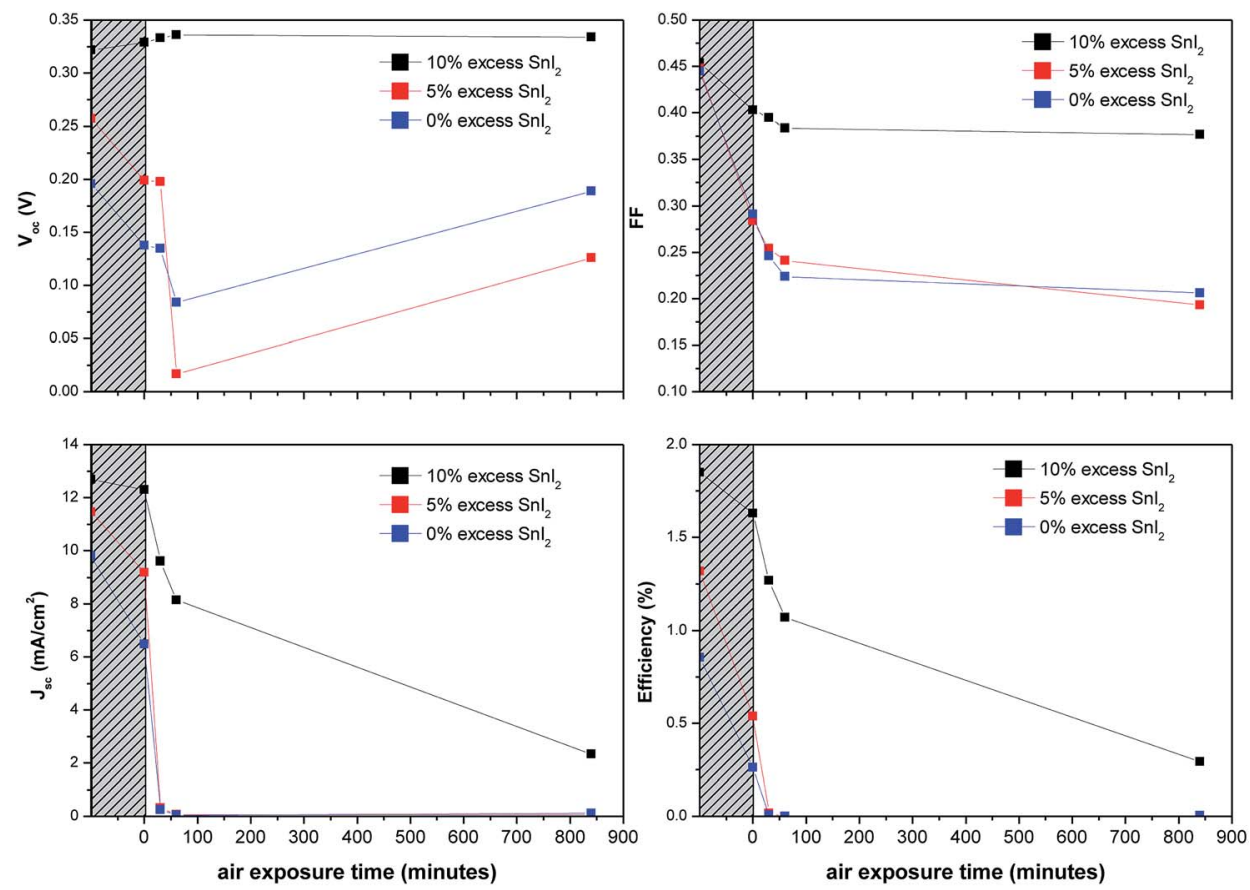

Fig. 6 Key device performance characteristics for unencapsulated PPV devices with the structure $|\mathrm{TO}| \mathrm{Cu}|| \mathrm{CsSn}{ }_{3}\left|\mathrm{C}_{60}\right| \mathrm{BCP} \mid \mathrm{Al}$ using $\left.\mathrm{CsSn}\right|_{3}$ prepared from solutions containing either 0,5 or $10 \mathrm{~mol} \%$ excess $\mathrm{Snl}_{2}$. The data in the hashed region correspond to device performance immediately after fabrication and then again after storage in a nitrogen atmosphere $\left(<5 \mathrm{ppm} \mathrm{O}_{2}\right.$ and $\left.<0.1 \mathrm{ppm} \mathrm{H}_{2} \mathrm{O}\right)$ for 10 days. These devices were then bought into laboratory and the performance was measured as a function of time stored in air. 

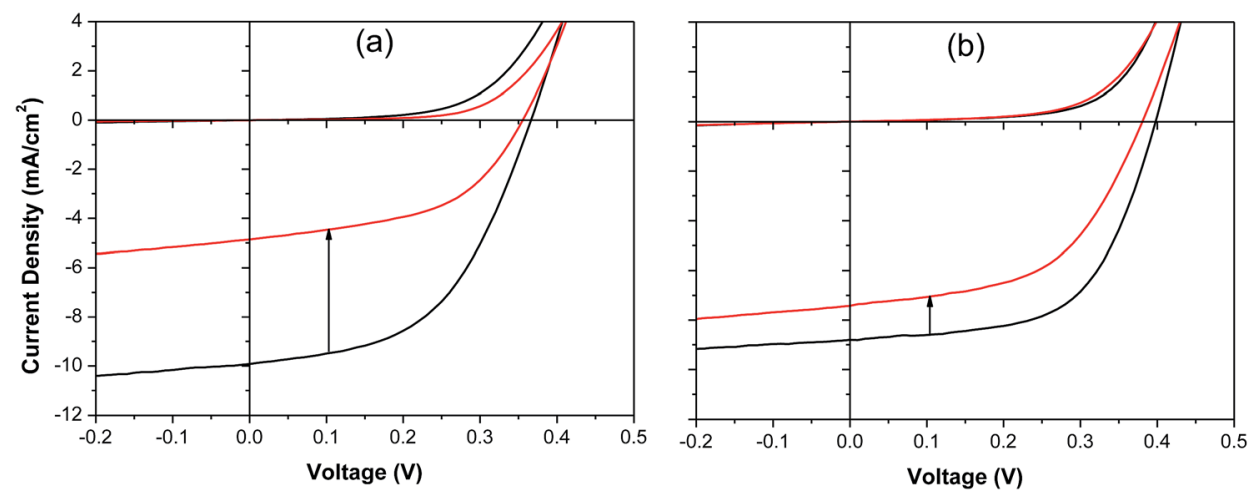

Fig. 7 Representative J-V characteristics of PPV devices with the structure: ITO|Cul $70 \mathrm{~nm} \mid \mathrm{CsSnl}_{3}$ (with and without 10 mol\% excess Snl ${ }_{2}$ ) IPCBM|BCP|Al: (a) $\mathrm{CsSnl}_{3}$ with no excess Snl 2 ; (b) $\mathrm{CsSnl}_{3}$ with 10 mol\% excess Snl $\mathrm{S}_{2}$. Graphs show devices made entirely in glove box (black) and those made using a CsSnl layer exposed to the laboratory environment for 1 minute (red) before being returned to the glove box to complete fabrication and testing.

continuous deterioration in $J_{\mathrm{sc}}$. This conclusion is supported by the evolution of the absorption spectra of $\mathrm{CsSnI}_{3}$ films on glass, with and without $10 \mathrm{~mol} \% \mathrm{SnI}_{2}$, as a function of time exposed to air ((ESI, Fig. $9 \dagger))$, from which it is evident that the addition of 10 mol\% $\mathrm{SnI}_{2}$ greatly reduces the rate of oxidation of the $\mathrm{CsSnI}_{3}$. Since this process would be expected to begin at the interface with the ETL any significant change in the energetics at this interface for electron extraction as a result of this transformation would manifest as a change in device series resistance and FF, the effect of which would be most pronounced in the early stages of oxidation process when the potential energy step forms. $\mathrm{Cs}_{2} \mathrm{SnI}_{6}$ has been shown to have a relatively high electron and hole mobility ${ }^{48}$ although its conduction band edge is $\sim 200 \mathrm{meV}$ deeper lying than that of $\mathrm{CsSnI}_{3}{ }^{41}$ and so the formation of a $\mathrm{Cs}_{2} \mathrm{SnI}_{6}$ layer between the remaining $\mathrm{CsSnI}_{3}$ and $\mathrm{C}_{60}$ would most likely impede the flow of electrons from the conduction band of the $\mathrm{CsSnI}_{3}$ into the LUMO of the $\mathrm{C}_{60}$ resulting in an increase in device series resistance and reduction in $\mathrm{FF}$, as is observed to be the case. To test the generality of this result for another fullerene ETL and to remove the complexity associated with possible doping of the fullerene layer by oxygen upon exposure to air we have fabricated PPV devices using PCBM as the ETL with and without exposure of the $\mathrm{CsSnI}_{3}$ layer to air for 1 minute, before transferring back into the glove box to complete device fabrication. Typical $J-V$ characteristics for PPV devices with and without exposure of the perovskite layer to air are given in Fig. 7 from
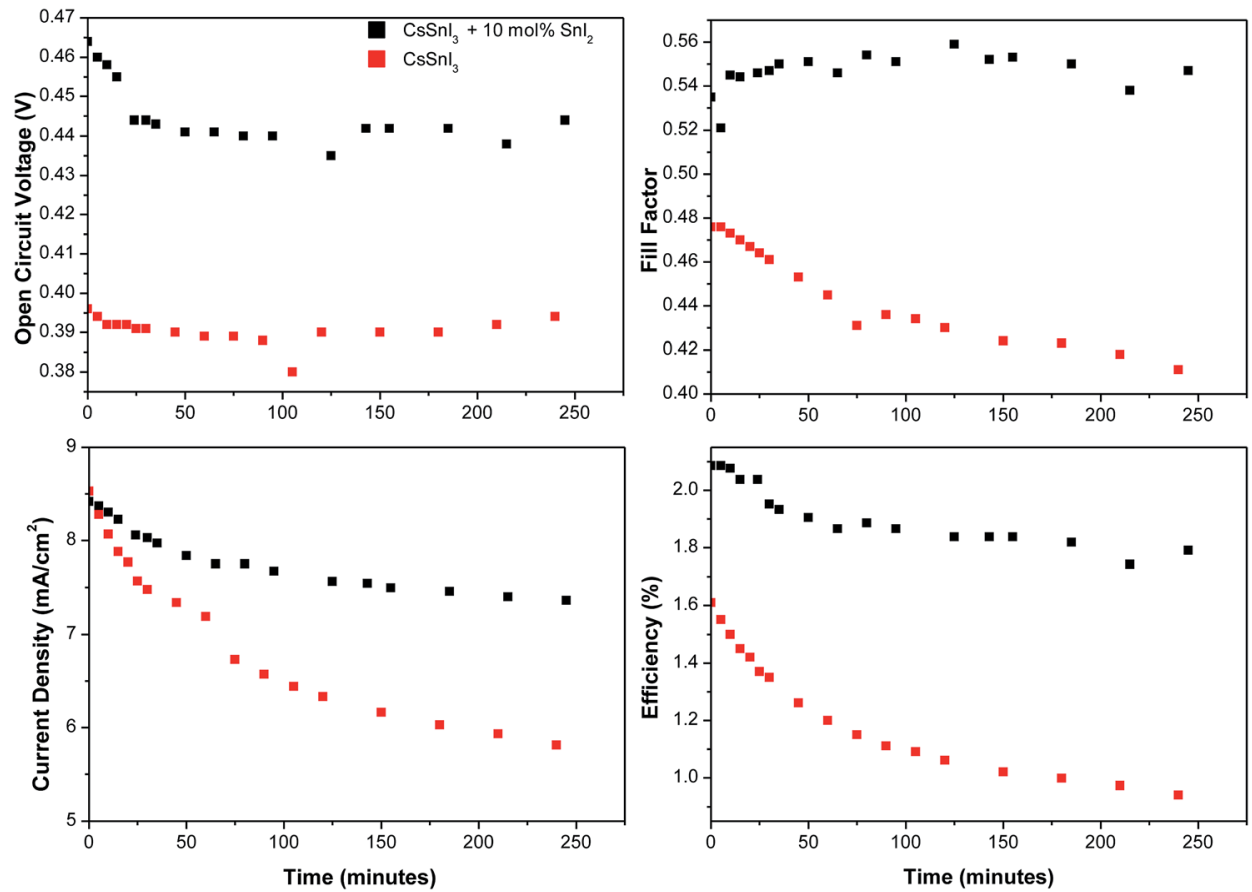

Fig. 8 Graphs showing the effect of constant illumination $\left(100 \mathrm{~mW} \mathrm{~cm}^{-2}\right.$ of AM 1.5) for 245 minutes. Device structure; ITO|Cul (70 $\left.\mathrm{nm}\right) \mid \mathrm{CsSnl} \mathrm{I}_{3}+$ $0 \%$ or $10 \% \mathrm{Snl}_{2}(8 \mathrm{wt} \%, 4000 \mathrm{rpm})\left|\mathrm{PC}{ }_{61} \mathrm{BM}\left(15 \mathrm{mg} \mathrm{ml}^{-1}, 1000 \mathrm{rpm}\right)\right| \mathrm{BCP}(8 \mathrm{~nm}) \mid \mathrm{Al}(50 \mathrm{~nm})$. 
which it is clear that devices incorporating an excess of $\mathrm{SnI}_{2}$ are much more stable than those without and that the changes in $J_{\mathrm{sc}}$, $V_{\mathrm{oc}}$ and $\mathrm{FF}$ after 1 minute air exposure are consistent with that in Fig. 6.

Finally, we have tested the stability of devices, with and without $10 \mathrm{~mol} \%$ excess $\mathrm{SnI}_{2}$ in the $\mathrm{CsSnI}_{3}$ layer, over a period of four hours continuous illumination in a nitrogen atmosphere. Representative results are given in Fig. 8, from which it is clear that devices incorporating excess $\mathrm{SnI}_{2}$ exhibit greatly improved stability including a stable $V_{\mathrm{oc}}$ and $\mathrm{FF}$ after the first 20 minutes. Whilst $J_{\mathrm{sc}}$ deteriorates rapidly by $\sim 10 \%$ within the first 50 minutes the rate of reduction in $J_{\mathrm{sc}}$ then slows markedly. Conversely devices with no excess of $\mathrm{CsSnI}_{3}$ exhibit a continuous rapid decline in FF and $J_{\mathrm{sc}}$.

Collectively the aforementioned stability studies show that the use of a $10 \mathrm{~mol} \%$ excess of $\mathrm{SnI}_{2}$ in $\mathrm{CsSnI}_{3}$ preparation imparts dramatically improved device stability, which indicates that the stability of $\mathrm{CsSnI}_{3}$ towards oxidation is very sensitive to the density of Sn vacancy defects. Since the transformation of $\mathrm{CsSnI}_{3}$ into $\mathrm{Cs}_{2} \mathrm{CsI}_{6}$ requires the loss of $\mathrm{Sn}$ it seems plausible that at the site of a Sn vacancy in $\mathrm{CsSnI}_{3}$ the barrier to transformation into $\mathrm{Cs}_{2} \mathrm{SnI}_{6}$ is reduced. It is also possible that the excess $\mathrm{SnI}_{2}$ occupies some of the space between adjacent $\mathrm{CsSnI}_{3}$ crystallites thereby impeding the ingress of the oxygen and water that gives rise to oxidation.

\section{Conclusion}

In summary we have demonstrated the preparation of orthorhombic $\mathrm{CsSnI}_{3}$ films from solution at room temperature with defect densities low enough for use as the light harvesting semiconductor in photovoltaic devices, even without the addition of excess Sn. We have shown how the $V_{\text {oc }}$ in a model planar layer device based on a $\mathrm{CuI} \mid \mathrm{CsSnI}_{3}$ |fullerene $\mathrm{p}-\mathrm{i}-\mathrm{n}$ structure can be doubled from $\sim 0.28 \mathrm{~V}$ to $\sim 0.55 \mathrm{~V}$ by engineering the energetics at the $\mathrm{CsSnI}_{3} /$ fullerene interface. To our knowledge, the champion device $V_{\mathrm{oc}}$, FF and $\eta$ are the highest that have been reported using $\mathrm{CsSnI}_{3}$ as the light harvesting semiconductor in a PPV by a considerable margin. We have also demonstrated a simple and effective strategy for improving the stability of PPV devices based on $\mathrm{CsSnI}_{3}$ and have rationalised the reason for this improvement using a combination of photoelectron spectroscopy, contact potential measurements and device based studies. This work shows that if tin-halide perovskites are prepared in a tin rich environment, in order to minimise the density of Sn vacancy defects, then consideration must be made as to the charge transport properties of the source of the excess tin.

\section{Materials}

CuI (Sigma-Aldrich, 99.999\%), CsI (Sigma-Aldrich 99.9\%), $\mathrm{SnI}_{2}$ (Alfa Aesar, 99.999\%), $\mathrm{PC}_{61} \mathrm{BM}$ (Nano-C, 99.5\%), indene- $\mathrm{C}_{60}$ bisadduct $\left(\mathrm{IC}_{60} \mathrm{BA}\right)$ (Ossila, 99.5\%), $\mathrm{C}_{60}$ (Nano-C, 99.5\%), bathocuproine (Alfa Aesar, 98\%), N,N-dimethylformamide (DMF) (VWR, anhydrous, 99.8\%), chlorobenzene (Sigma-Aldrich, anhydrous, 99.8\%), acetone (Sigma-Aldrich, GPR, $\geq 99 \%$ ), propan-2-ol (Sigma-Aldrich, HPLC, 99.8\%), deionised $\mathrm{H}_{2} \mathrm{O}$ (pureit dispenser, $>10 \mathrm{M} \Omega$ ). CsI and non-anhydrous solvents were stored in air. All other chemicals were stored in nitrogen filled glove box $\left(<5 \mathrm{ppm} \mathrm{O}_{2}\right.$ and $\left.<0.1 \mathrm{ppm}_{2} \mathrm{O}\right)$.

\section{Synthesis of caesium tin(II) iodide}

CsI was added to $\mathrm{SnI}_{2}$ in a $1: 1.1$ molar ratio under nitrogen. To this DMF was added to make an $8 \mathrm{wt} \%$ solution which is bright yellow in colour. The solution was shaken for a few minutes until all CsI and $\mathrm{SnI}_{2}$ had dissolved and was left overnight before use. The perovskite material precipitated on spin casting to give dark red/brown coloured films.

\section{Device fabrication}

Solar cells were fabricated on indium tin oxide (ITO) coated glass slides (Thin Film Devices Inc., $15 \pm 3 \Omega \mathrm{sq}^{-1}$ ). The slides were prepared by cleaning the ITO using acetone, water and isopropanol in an ultrasound bath for 10 minutes each followed by $\mathrm{UV} / \mathrm{O}_{3}$ treatment for 15 minutes. The slides were then loaded into a dry nitrogen filled glove box for device fabrication. 70-100 $\mathrm{nm}$ thick $\mathrm{CuI}$ films were deposited onto the ITO glass substrates by thermal evaporation. CuI can also be deposited by spin casting a $50 \mathrm{mg} \mathrm{ml}^{-1}$ in chlorobenzene : di- $n$-propyl sulfide $(5.5: 1)$ at $1000 \mathrm{rpm}$. Onto this $8 \mathrm{wt} \% \mathrm{CsSnI}_{3}$ with a $10 \mathrm{~mol} \%$ excess of $\mathrm{SnI}_{2}$ was spin cast at $4000 \mathrm{rpm}$ from a DMF solution for $60 \mathrm{~s} .40 \mathrm{~nm}$ of $\mathrm{C}_{60}$ was the deposited by thermal evaporation at a rate of $0.1-0.4 \mathrm{~nm} \mathrm{~s}^{-1} \cdot \mathrm{PC}_{61} \mathrm{BM}$ was spin cast at $1000 \mathrm{rpm}$ from $15 \mathrm{mg} \mathrm{ml}^{-1}$ chlorobenzene solution, and $\mathrm{IC}_{60} \mathrm{BA}$ from 3, 5 or $7 \mathrm{mg} \mathrm{ml}^{-1}$ chlorobenzene solutions for $60 \mathrm{~s}$. The PCBM and ICBA solutions were filtered with a $0.2 \mu \mathrm{m}$ PTFE syringe filter before use. Finally $8 \mathrm{~nm}$ of bathocuproine (BCP) followed by 50 $\mathrm{nm}$ of $\mathrm{Al}$ were deposited by thermal evaporation at a rate of $0.5-$ $1 \AA \mathrm{s}^{-1}$. The $\mathrm{Al}$ was evaporated through a mask to produce 0.06 $\mathrm{cm}^{2}$ pixels. For devices without CuI, ITO was cleaned in the same way, but was put under the $\mathrm{UV} / \mathrm{O}_{3}$ lamp for 5 minutes longer to help the perovskite film wet the surface.

\section{Device testing}

Device testing was performed in the same glove box as used for device fabrication unless otherwise stated. Current-voltage $(J-V)$ curves were measured using a Keithley 2400 source-meter under AM1.5G solar illumination at $100 \mathrm{~mW} \mathrm{~cm}^{-2}$ (1 sun) by sweeping from $-1 \mathrm{~V}$ to $+1 \mathrm{~V}$ in $0.01 \mathrm{~V}$ increments at a rate of $0.1 \mathrm{~V} \mathrm{~s}^{-1}$. There was no significant current-voltage hysteresis, as shown in Fig. S10. $\dagger$ IPCE measurements were carried out using a Sciencetech SF150 xenon arc lamp and a PTI monochromator, with the monochromatic light intensity calibrated using a Si photodiode (Newport 818-UV). The incoming monochromatic light was chopped at $500 \mathrm{~Hz}$. For signal measurement a Stanford Research Systems SR 830 lock-in amplifier was used.

\section{X-ray diffraction}

$\mathrm{CsSnI}_{3}$ films were spin cast from $16 \mathrm{wt} \%$ DMF solutions at 2000 rpm onto glass slides coated with $10 \mathrm{~nm}$ of CuI. The X-ray 
diffraction patterns were collected under a flow of nitrogen using a $\mathrm{Cu} \mathrm{K} \alpha_{1 / 2}$ source in $\theta-\theta$ mode on a Bruker D8 Advance powder diffractometer equipped with an Anton-Paar HTK900 gas chamber. Patterns were corrected for the height of the sample by calibrating the $2 \theta$ axis against reference patterns. Simulated diffraction patterns were calculated using the program Mercury 3.1 with CIFs from the Inorganic Crystal Structure Database (ICSD).

\section{UPS and XPS}

UPS and XPS were performed on freshly prepared $\mathrm{CsSnI}_{3}$ films spin cast onto Au substrates. The samples were exposed to the laboratory environment for $\sim 1$ min prior to loading into the ultra-high vacuum system. UPS spectra were recorded using UV photons with energy $21.22 \mathrm{eV}$ from a He I plasma source. The Xray photoelectron spectroscopy (XPS) measurements were carried out in a UHV system with a base pressure of $5 \times 10^{-11}$ mbar. The sample was excited with X-rays from a mono-chromated $\mathrm{Al} \mathrm{K} \alpha$ source $(h \nu=1486.6 \mathrm{eV})$ with the photoelectrons being detected at a $45^{\circ}$ take-off angle using an Omicron Sphera electron analyser.

\section{Acknowledgements}

This work was supported by the UK Engineering and Physical Science Research Council and European Regional Development Agency/Advantage West Midlands Science City Materials Initiative (Project 2). We thank Dr Marc Walker (University of Warwick, Department of Physics) for assistance with the XPS and UPS data collection.

\section{Notes and references}

1 A. Kojima, K. Teshima, Y. Shirai and T. Miyasaka, J. Am. Chem. Soc., 2009, 131, 6050-6051.

2 J. M. Ball, M. M. Lee, A. Hey and H. J. Snaith, Energy Environ. Sci., 2013, 6, 1739-1743.

3 B. Cai, Y. Xing, Z. Yang, W.-H. Zhang and J. Qiu, Energy Environ. Sci., 2013, 6, 1480-1485.

4 L. Etgar, P. Gao, Z. Xue, Q. Peng, A. K. Chandiran, B. Liu, M. K. Nazeeruddin and M. Grätzel, J. Am. Chem. Soc., 2012, 134, 17396-17399.

5 S. Colella, E. Mosconi, P. Fedeli, A. Listorti, F. Gazza, F. Orlandi, P. Ferro, T. Besagni, A. Rizzo, G. Calestani, G. Gigli, F. De Angelis and R. Mosca, Chem. Mater., 2013, 25, 4613-4618.

6 M. M. Lee, J. Teuscher, T. Miyasaka, T. N. Murakami and H. J. Snaith, Science, 2012, 338, 643-647.

7 M. Liu, M. B. Johnston and H. J. Snaith, Nature, 2013, 501, 395-398.

8 S. D. Stranks, G. E. Eperon, G. Grancini, C. Menelaou, M. J. P. Alcocer, T. Leijtens, L. M. Herz, A. Petrozza and H. J. Snaith, Science, 2013, 342, 341-344.

9 H.-S. Kim, C.-R. Lee, J.-H. Im, K.-B. Lee, T. Moehl, A. Marchioro, S.-J. Moon, R. Humphry-Baker, J.-H. Yum, J. E. Moser, M. Grätzel and N.-G. Park, Sci. Rep., 2012, 2, 591.
10 H. Zhou, Q. Chen, G. Li, S. Luo, T.-B. Song, H.-S. Duan, Z. Hong, J. You, Y. Liu and Y. Yang, Science, 2014, 345, 542-546.

11 J.-Y. Jeng, Y.-F. Chiang, M.-H. Lee, S.-R. Peng, T.-F. Guo, P. Chen and T.-C. Wen, Adv. Mater., 2013, 25, 3727-3732.

12 C. Zuo and L. Ding, Nanoscale, 2014, 6, 9935-9938.

13 W. Nie, H. Tsai, R. Asadpour, A. J. Neukirch, G. Gupta, J. J. Crochet, M. Chhowalla, S. Tretiak, M. A. Alam and H. Wang, Science, 2015, 347, 522-525.

14 I. Chung, J.-H. Song, J. Im, J. Androulakis, C. D. Malliakas, H. Li, A. J. Freeman, J. T. Kenney and M. G. Kanatzidis, J. Am. Chem. Soc., 2012, 134, 8579-8587.

15 J. A. Christians, R. C. M. Fung and P. V. Kamat, J. Am. Chem. Soc., 2014, 136, 758-764.

16 N. J. Jeon, J. Lee, J. H. Noh, M. K. Nazeeruddin, M. Grätzel and S. Il Seok, J. Am. Chem. Soc., 2013, 135, 19087-19090.

17 J. Wang, S. Wang, X. Li, L. Zhu, Q. Meng, Y. Xiao and D. Li, Chem. Commun., 2014, 50, 5829-5832.

18 A. S. Subbiah, A. Halder, S. Ghosh, N. Mahuli, G. Hodes and S. K. Sarkar, J. Phys. Chem. Lett., 2014, 5, 1748-1753.

19 G. Niu, W. Li, F. Meng, L. Wang, H. Dong and Y. Qiu, J. Mater. Chem. A, 2014, 2, 705.

20 M. A. Green, A. Ho-Baillie and H. J. Snaith, Nat. Photonics, 2014, 8, 506-514.

21 M. Grätzel, Nat. Mater., 2014, 13, 838-842.

22 H. L. Clever and F. J. Johnston, J. Phys. Chem. Ref. Data, 1980, 9, 751-784.

23 F. Bischoff, L. C. Maxwell, R. D. Evans and F. R. Nuzum, J. Pharmacol. Exp. Ther., 1928, 34, 85-109.

24 Y. Ogomi, A. Morita, S. Tsukamoto, T. Saitho, N. Fujikawa, Q. Shen, T. Toyoda, K. Yoshino, S. S. Pandey and S. Hayase, J. Phys. Chem. Lett., 2014, 5, 1004-1011.

25 I. Chung, B. Lee, J. He, R. P. H. Chang and M. G. Kanatzidis, Nature, 2012, 485, 486-489.

26 Z. Chen, J. J. Wang, Y. Ren, C. Yu and K. Shum, Appl. Phys. Lett., 2012, 101, 093901.

27 M. H. Kumar, S. Dharani, W. L. Leong, P. P. Boix, R. R. Prabhakar, T. Baikie, C. Shi, H. Ding, R. Ramesh, M. Asta, M. Graetzel, S. G. Mhaisalkar and N. Mathews, Adv. Mater., 2014, 26, 7122-7127.

28 D. Sabba, H. K. Mulmudi and R. R. Prabhakar, J. Phys. Chem. C, 2015, 119, 1763-1767.

29 N. K. Noel, S. D. Stranks, A. Abate, C. Wehrenfennig, S. Guarnera, A. Haghighirad, A. Sadhanala, G. E. Eperon, S. K. Pathak, M. B. Johnston, A. Petrozza, L. Herz and H. Snaith, Energy Environ. Sci., 2014, 7, 3061-3068.

30 F. Hao, C. C. Stoumpos, D. H. Cao, R. P. H. Chang and M. G. Kanatzidis, Nat. Photonics, 2014, 8, 489-494.

31 W. Shockley and H. J. Queisser, J. Appl. Phys., 1961, 32, 510519.

32 K. Shum, Z. Chen, J. Qureshi, C. Yu, J. J. Wang, W. Pfenninger, N. Vockic, J. Midgley and J. T. Kenney, Appl. Phys. Lett., 2010, 96, 221903.

33 Z. Chen, C. Yu, K. Shum, J. J. Wang, W. Pfenninger, N. Vockic, J. Midgley and J. T. Kenney, J. Lumin., 2012, 132, 345-349. 
34 P. Xu, S. Chen, H. Xiang, X. Gong and S. Wei, Chem. Mater., 2014, 26, 6068-6072.

35 C. C. Stoumpos, C. D. Malliakas and M. G. Kanatzidis, Inorg. Chem., 2013, 52, 9019-9038.

36 U. Kaldor and S. Wilson, Theoretical Chemistry and Physics of Heavy and Superheavy Elements, 2003.

37 P. Pyykkö, Chem. Rev., 1988, 88, 563-594.

38 Y. Zhou, H. F. Garces, B. S. Senturk, A. L. Ortiz and N. P. Padture, Mater. Lett., 2013, 110, 127-129.

39 W. Werker, Recl. Trav. Chim. Pays-Bas Belg., 1939, 58, 257-258.

40 T. G. Plint, B. a. Kamino and T. P. Bender, J. Phys. Chem. C, 2015, 119, 1676-1682.

41 J. Zhang, C. Yu, L. Wang, Y. Li, Y. Ren and K. Shum, Sci. Rep., 2014, 4, 6954.

42 B. H. Ishii, K. Sugiyama, E. Ito and K. Seki, Adv. Mater., 1999, 11, 605-625.
43 S. R. Day, R. A. Hatton, M. A. Chesters and M. R. Willis, Thin Solid Films, 2002, 410, 159-166.

44 P. Ravindran, A. Delin, R. Ahuja, B. Johansson, S. Auluck, J. Wills and O. Eriksson, Phys. Rev. B: Condens. Matter Mater. Phys., 1997, 56, 6851-6861.

45 B. W. Larson, J. B. Whitaker, X. Wang, A. A. Popov, G. Rumbles, N. Kopidakis, S. H. Strauss and O. V. Boltalina, J. Phys. Chem. C, 2013, 117, 14958-14964.

46 Y. He, H. Y. Chen, J. Hou and Y. Li, J. Am. Chem. Soc., 2010, 132, 1377-1382.

47 N. Grossiord, J. M. Kroon, R. Andriessen and P. W. M. Blom, Org. Electron., 2012, 13, 432-456.

48 B. Lee, C. C. Stoumpos, N. Zhou, F. Hao, C. Malliakas, C. Yeh, T. J. Marks, M. G. Kanatzidis and R. P. H. Chang, J. Am. Chem. Soc., 2014, 136, 15379-15385. 